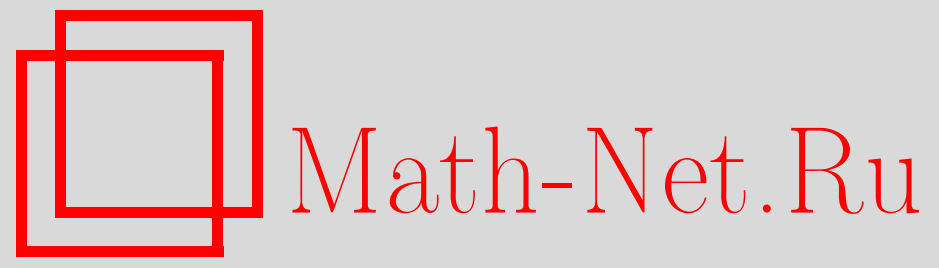

А. Н. Дегтев, Об обобщениях понятия полурекурсивности, Дискрет. матем., 2005, том 17, выпуск 2, 144-149

DOI: https://doi.org/10.4213/dm106

Использование Общероссийского математического портала Math-Net.Ru подразумевает, что вы прочитали и согласны с пользовательским соглашением http://www.mathnet.ru/rus/agreement

Параметры загрузки:

IP: 54.197 .217 .227

26 апреля 2023 г., $17: 28: 33$

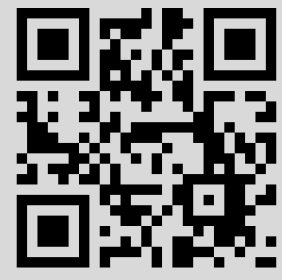




\title{
Об обобщениях понятия полурекурсивности
}

\author{
(ㄷ) 2005 г. А. Н. Дегтев
}

Наравне с классами полурекурсивных, слабо полурекурсивных, полурекурсивноперечислимых и wsז* множеств, введенных К. Джокушем, М. Куммером и Ф. Стефаном, определяются классы почти и частично полурекурсивных множеств. Полностью выясняются соотношения между этими классами множеств относительно включения.

Пусть $A \subseteq \mathbf{N}=\{0,1,2, \ldots\}$ и существует двуместная общерекурсивная функщия $f$ такая, что для всех $x, y \in \mathbf{N}$ выполнено одно из условий

$$
\begin{aligned}
x \in A \vee y \in A & \Longleftrightarrow f(x, y) \in\{x, y\} \cap A, \\
x \in A \wedge y \in A & \Longleftrightarrow f(x, y) \in\{x, y\} \cap A, \\
(x \in A \wedge y \notin A) \vee(x \notin A \wedge y \in A) & \Longrightarrow f(x, y) \in\{x, y\} \cap A .
\end{aligned}
$$

Тогда множество $A$ называется полурекурсивным. Несложно показать $[7,9]$, что эти три условия равносильны. Понятие полурекурсивности неоднократно обобщалось $[1-6,8]$.

Множество $A \subseteq \mathbf{N}$ называется

- слабо полурекурсивным (wsr множеством) [7], если найдется двуместная частично рекурсивная функщия $f$ такая, что для всех $x, y \in \mathbf{N}$ выполнено условие (1),

- полурекурсивно-перечислимым (sre множеством), если

$$
x \in A \vee y \in A \Longrightarrow f(x, y) \in\{x, y\} \cap A,
$$

- wsr* множеством [9], если выполнены условия

(a) $f(x, y) \downarrow \Longrightarrow f(x, y) \in\{x, y\}$,

(b) $x \in A \wedge y \in A \Longrightarrow f(x, y) \downarrow$,

(c) $f(x, y) \downarrow \wedge f(x, y) \in A \Longrightarrow x \in A \wedge y \in A$,

где $f(x, y) \downarrow$ означает, что значение $f(x, y)$ определено.

С точки зрения определений слабо $\beta$-комбинаторно селекторных [3] и слабо $\beta$-импликативно селекторных множеств [4], wsr* и sre множества будут соответственно слабо $x y$-комбинаторно селекторным и $x \vee y$-комбинаторно селекторным множествами, a wsr множество - слабо ( $\bar{y} x \vee \bar{x} y)$-импликативно селекторным множеством. Понятия слабо $x \wedge y$-импликативно селекторных и $x \vee y$-комбинаторно селекторных множеств совпадают. Но можно ввести в рассмотрение класс слабо $x y$-импликативно селекторных множеств $A$, 
определяемых следующим образом (см. [4]): существует двуместная частично рекурсивная функция $f$ такая, что для всех $x, y \in \mathbf{N}$

$$
x \in A \wedge y \in A \Longrightarrow f(x, y) \in\{x, y\} \cap A .
$$

Однако нетрудно понять, что класс таких множеств совпадает с $F^{\infty}$ в обозначениях статьи [4], то есть с классом всех подмножеств $\mathbf{N}$.

Пусть $A \subseteq \mathbf{N} \bar{a} \in \bar{A}=\mathbf{N} \backslash A$ и $\beta-n$-местная булева функция. Тогда $A$ было названо в [6] почти $\beta$-комбинаторно селекторным множеством, если существует $n$-местная общерекурсивная функщия $f$ такая, что для всех $x_{1}, \ldots, x_{n} \in \mathbf{N}$

$$
\begin{aligned}
\beta\left(\chi\left(x_{1}\right), \ldots, \chi\left(x_{n}\right)\right)=1 \Longleftrightarrow f\left(x_{1}, \ldots, x_{n}\right) \in\left\{x_{1}, \ldots, x_{n}, \bar{a}\right\} \cap A, \\
\\
\quad f\left(x_{1}, \ldots, x_{n}\right) \in\left\{x_{1}, \ldots, x_{n}, \bar{a}\right\},
\end{aligned}
$$

где $\chi$ - характеристическая функция $A$. При $\beta=x y$ назовем $A$ почти полурекурсивным (asr) множеством. Если в (2) $\bar{a}$ заменено на $a \in A$, то $A$ называется частично $\beta$-комбинаторно селекторным множеством, а при $\beta=x \vee y$ - частично полурекурсивным (psr) множеством. Если же в (2) выбросить $\bar{a}$, то придем к понятию $\beta$-селекторного множества [1]. Для них в [1] получен следующий результат: если булева функция $\beta$ такова, что $\beta(x, \ldots, x)=x$, то класс всех $\beta$-селекторных множеств совпадает с классом $F^{(0)}$ всех рекурсивных множеств, или с классом $F^{(1)}$ всех полурекурсивных множеств, или с классом $F^{(\infty)}$.

Выше в определениях различных понятий множества $A$ участвовала общерекурсивная функция или частично рекурсивная функция $f$. Будем говорить, что $A$ является одним из вышеназванных множеств посредством $f$, а $f$ называть функцией, соответствующей $A$.

Предложение 1. Каждое множество, являющееся одновременно asr $u$ wsr множеством, полурекурсивно.

Доказательство. Каждое asr множество $A$ является wsr* множеством, поэтому, будучи и wsr множеством, $A$ является sre множеством [10]. Поэтому пусть $A$ - asr и sre множество посредством общерекурсивной функции $f$ и частично рекурсивной функции $g$ соответственно и

$$
\begin{aligned}
B_{0} & =\{x:(\exists y)(f(x, y)=\bar{a} \wedge g(x, y)=y)\}, \\
B_{n+1} & =\left\{x:(\exists y)\left(y \in B_{n} \wedge f(x, y)=x\right)\right\}, \quad n=0,1, \ldots
\end{aligned}
$$

Заметим, что $B_{0} \neq \varnothing$, так как если $y \in A$, то $g(\bar{a}, y)=y$ и, следовательно, $\bar{a} \in A$. Далее, $B=\bigcup_{n \geqslant 0} B_{n}$ - рекурсивно перечислимое подмножество $\bar{A}$, и если $\bar{A}=B$, то $A$ - asr множество с рекурсивно перечислимым дополнением, а поэтому полурекурсивно. Действительно, определим общерекурсивную функщию $h$ следующим образом. Если $f(x, y) \in\{x, y\}$, то положим $h(x, y)=f(x, y)$. Если же $f(x, y)=\bar{a}$, то по крайней мере один из элементов $x, y$ принадлежит $\bar{A}$. Перечисляя элементы множества $A$, обнаружим такое число и положим $h(x, y)$ равным этому числу. Ясно, что $A$ оказалось $x y$-селекторным множеством посредством общерекурсивной функции $h$, а поэтому и полурекурсивным.

Пусть теперь $b \in A \backslash B$ и $R=\{y: f(b, y)=y\}$. Тогда $R$ - рекурсивное множество, $B \subseteq R \subseteq A$ и

$$
x \in \bar{R} \wedge f(x, y)=\bar{a} \Longrightarrow y \in \bar{A} .
$$


Действительно, если $y \in A$, то $x \in \bar{A}$ и $g(x, y)=y$. Поэтому $x \in B_{0}$, что противоречит предположению. Теперь положим по определению

$$
h(x, y)= \begin{cases}f(x, y), & \text { если } f(x, y) \in\{x, y\} \wedge x \in \bar{R}, \\ x, & \text { если } x \in R \\ y, & \text { если } x \in \bar{R} \wedge f(x, y)=a .\end{cases}
$$

Легко проверить, что $A$ является $x y$-селекторным множеством посредством общерекурсивной функции $h$ и, следовательно, полурекурсивным множеством.

Обозначим через $K(\mathrm{abc})$ класс всех abc множеств.

Теорема 1. Справедливы следующие соотночения по включению между классами wsr, wsr*, sre, asr $u$ sr множеств:

$$
\begin{aligned}
K(\mathrm{asr}) & \subseteq K\left(\mathrm{wsr}^{*}\right), \\
K(\mathrm{sr}) & =K(\mathrm{sre}) \cap K(\mathrm{asr}), \\
K(\mathrm{sre}) & =K(\mathrm{wsr}) \cap K\left(\mathrm{wsr}^{*}\right), \\
K\left(\mathrm{wsr}^{*}\right) & \backslash K(\mathrm{wsr}) \cup K(\mathrm{asr}) \neq \varnothing, \\
K(\mathrm{asr}) & \backslash K(\mathrm{wsr}) \neq \varnothing, \\
K(\mathrm{wsr}) & \backslash K\left(\mathrm{wsr}{ }^{*}\right) \neq \varnothing, \\
K(\mathrm{sre}) & \backslash K(\mathrm{sr}) \neq \varnothing .
\end{aligned}
$$

Доказательство. Первое включение очевидно, ясно также, что каждое полурекурсивное sr множество является как и sre, так и asr множеством, а по предложению 1 верно и обратное. В то же время $A$ является sre множеством тогда и только тогда, когда $A-$ одновременно wsr и wsr* множество [10]. С другой стороны, существуют wsr* множества, не являющиеся wsr или asr множествами, так же как и asr, но не wsr множества [5]. Легко показать также, что $A$ полурекурсивно тогда и только тогда, когда $A$ и $\bar{A}$ - sre множества [9]. Значит, если $A$ - частично полурекурсивное, но не полурекурсивное множество, то $\bar{A}$ будет wsr, но не wsr* множеством [9], а само $A$ будет не полурекурсивным sre множеством.

Обратимся теперь к psr множествам. Ясно, что если $A$ - asr множество посредством общерекурсивной функщии $f$, то $\bar{A}-$ psr множество посредством той же общерекурсивной функции $f$. Поэтому из предложения 1 вытекает следующее утверждение.

Следствие 1. Каждое psr множество с wsr дополнением является полурекурсивным.

Предложение 2. Каждое множество, являющееся одновременно psr $u$ wsr множеством, полурекурсивно.

Доказательство. Пусть $A$ - psr и wsr множество посредством общерекурсивной функщии $f$ и частично рекурсивной функции $g$ соответственно и

$$
\begin{aligned}
B_{0} & =\{g(x, y): f(x, y)=a\}, \\
B_{n+1} & =\left\{x:(\exists y)\left(y \in B_{n} \wedge f(x, y)=x\right)\right\}, \quad n=0,1,2, \ldots
\end{aligned}
$$

Можно считать, что $B_{0} \neq \varnothing$, так как в противном случае $f(x, y)=a \Longrightarrow x, y \in A$, и полурекурсивность $A$ очевидна. Ясно также, что $B=\bigcup_{n \geqslant 0} B_{n}$ - рекурсивно перечислимое подмножество $A$, и если $A=B$, то $\bar{A}$ будет asr множеством с рекурсивно 
перечислимым дополнением и, как показано в предложении $1, A$ оказывается полурекурсивным множеством.

Пусть теперь $b \in A \backslash B$ и $R=\{y: f(b, y)=y\}$. Значит, $R$ - рекурсивное множество, $B \subseteq R \subseteq A$ и

$$
x \in \bar{R} \wedge f(x, y)=a \Longrightarrow y \in A,
$$

так как $y \in \bar{A} \Longrightarrow x \in A \Longrightarrow g(x, y)=x \Longrightarrow x \in B_{0}$, что противоречит выбору $x$. Положим по определению

$$
h(x, y)= \begin{cases}f(x, y), & \text { если } f(x, y) \in\{x, y\} \wedge x \in \bar{R}, \\ x, & \text { если } x \in R, \\ y, & \text { если } f(x, y)=a \wedge x \in \bar{R} .\end{cases}
$$

Понятно, что $A$ является полурекурсивным множеством посредством общерекурсивной функщии $h$.

Предложение 3. Каждое множество, являющееся одновременно $\mathrm{psr} u \mathrm{wsr}^{*}$ множеством, полурекурсивно.

Доказательство. Пусть $A$ - psr и wsr множество посредством общерекурсивной функцин $f$ и частично рекурсивной функции $g$ соответственно и

$$
\begin{aligned}
B_{0} & =\{x:(\exists y)(f(x, y)=a \wedge g(x, y)=y)\}, \\
B_{n+1} & =\left\{x:(\exists y)\left(f(x, y)=x \wedge y \in B_{n}\right)\right\}, \quad n=0,1,2, \ldots
\end{aligned}
$$

Можно считать, что $g(a, a)=a$ и так как $f(a, a)=a$, то $a \in B_{0}$, то есть $B_{0} \neq \varnothing$. Ясно, что $B=\bigcup_{\geqslant 0} B_{n}$ - рекурсивно перечислимое подмножество $A$, и если $B=A$, то $A$, как и в предложении 1 , оказывается полурекурсивным множеством.

Пусть теперь $b \in A \backslash B$ и $R=\{y: f(b, y)=y\}$. Понятно также, что $R \subseteq A$ и $y \in B \Rightarrow f(b, y)=y$, так как в противном случае $b \in B$, что противоречит выбору элемента $b$. Значит, $R$ - рекурсивное множество и $B \subseteq R \subseteq A$.

Предположим, что для всех $x, y \in \mathbf{N}$, если $f(x, y)=a$, то $\{x, y\} \cap A \neq \varnothing$. Тогда $A-$ полурекурсивное множество посредством общерекурсивной функщии $h$, где

$$
h(x, y)= \begin{cases}f(x, y), & \text { если } f(x, y) \in\{x, y\}, \\ x, & \text { если } f(x, y)=a \wedge x \in R, \\ y, & \text { если } f(x, y)=a \wedge y \in R .\end{cases}
$$

Если же $f(x, y)=a$ и $\{x, y\} \cap R=\varnothing$, то $g(x, y) \uparrow$, так как в противном случае либо $x$, либо $y$ принадлежал бы $B_{0}$ и поэтому принадлежал бы и $R$, что противоречит предположению. Следовательно, в этом случае один элемент из $x, y$ принадлежит $\bar{A}$. Пусть $\bar{a}$ - фиксированный элемент из $\bar{A}$. Тогда $A$ будет asr множеством посредством общерекурсивной функции $h$, где

$$
h(x, y)= \begin{cases}f(x, y), & \text { если } f(x, y)=y \vee(f(x, y)=a \wedge y \in R), \\ x, & \text { если } f(x, y)=x \vee(f(x, y)=a \wedge x \in R), \\ a, & \text { если } f(x, y)=a \vee\{x, y\} \cap R=\varnothing .\end{cases}
$$

Но $\bar{A}$ - также asr множество, поэтому $A$ - полурекурсивное множество (см. [5]). 
Теорема 2. Каждое psr множество, которое является wsr или wsr' $^{*}$ множеством, полурекурсивно, но существует psr множество, которое не является ни wsr, ни шsr* множеством.

Доказательство. Первая часть утверждения теоремы следует из предложений 2 и 3. Докажем вторую часть.

B [5] автором было построено asr множество, не являющееся wsr множеством и тем более полурекурсивным. Тогда дополнение этого множества будет psr, но по предложениям 2 и 3 ни wsr, ни wsr* множеством.

Предложение 4. Каждое почти $\beta$-комбинаторно селекторное множество является частично $\gamma$-комбинаторно селекторным множеством для подходящей булевой функции $\gamma$.

Доказательство. Пусть $\bar{A}$ - почти $\beta$-комбинаторно селекторное множество посредством общерекурсивной функции $f, \bar{a} \in \bar{A}$ и булева функци $\gamma$ определена следующим образом: $\gamma\left(\theta_{1}, \ldots, \theta_{n}\right)=\bar{\beta}\left(\dot{\theta}_{1}, \ldots, \bar{\theta}_{n}\right)$.

Справедливы соотношения

$$
\begin{aligned}
\beta\left(\chi\left(x_{1}\right), \ldots,\left(x_{n}\right)\right)=1 & \Rightarrow f\left(x_{1}, \ldots, x_{n}\right) \in\left\{x_{1}, \ldots, x_{n}\right\} \cap A \\
& \Longrightarrow \gamma\left(\overline{\chi\left(\overline{x_{1}}\right)}, \ldots, \overline{\chi\left(\overline{x_{n}}\right)}\right)=1, \\
\beta\left(\chi\left(x_{1}\right), \ldots, \chi\left(x_{n}\right)\right)=0 & \Longrightarrow\left(f\left(x_{1}, \ldots, x_{n}\right) \in \cap A\right) \vee\left(f\left(x_{1}, \ldots, x_{n}\right)=a\right) \\
& \Longrightarrow \gamma\left(\overline{\chi\left(\overline{x_{1}}\right)}, \ldots, \overline{\chi\left(\overline{x_{n}}\right)}\right)=1 .
\end{aligned}
$$

Поэтому $A$ будет psr множеством посредством общерекурсивной функции $f$.

Пусть $P(\beta)$ - класс всех частично $\beta$-комбинаторно селекторных множеств.

Теорема 3. Если булева функиия $\beta$ такова, что $\beta(x, \ldots, x)=x$, то $P(\beta)$ совпадает $c$ одним из классов $F^{(0)} \subset F^{(1)} \subset P(x \vee y) \subset F^{\infty}$, причем все включения строгие.

Доказательство. В [6] автором было доказано, что если булева функция $\beta$ такова, что $\beta(x, \ldots, x)=x$, то класс $A(\beta)$ всех почти $\beta$-комбинаторно селекторных множеств совпадает с одним из классов $F^{(0)} \subset F^{(1)} \subset A(x y) \subset F^{\infty}$, причем все включения строгие. Используя предложение 4 , получаем утверждение теоремы. Напомним, что классы $A(x y)$ и $P(x \vee y)$ являются классами почти и частично полурекурсивных множеств соответственно.

В заключение дадим ответ на вопрос из работы [3]. Пусть $\beta=x \vee y$ и $\gamma=$ $x y \vee x z \vee y z$, а $K(\beta)$ и $K(\gamma)$ - соответственно классы слабо $\beta$-комбинаторно селекторных и $\gamma$-комбинаторно селекторных множеств. В [3] показано, что $K(\beta) \subseteq K(\gamma)$, но оставалось не ясным, является ли это включение строгим. Оказывается, имеет место следующая теорема.

Теорема 4. Справедливо равенство $K(\beta)=K(\gamma)$.

Доказательство. Пусть $A \in K(\gamma)$, трехместная частично рекурсивная функция $f$ соответствует $A$ и $a, \bar{a}-$ фиксированные элементы из $A$ и $\bar{A}$. Будем определять двуместную частично рекурсивную функцию $g$, посредством которой $A$ окажется слабо $\beta$-комбинаторно селекторным множеством. Ясно, что если $x \in A$ или $y \in A$, то $f(x, y, a) \downarrow$. Поэтому начинаем вычислять значение $f(x, y, a)$, и если $f(x, y, a) \uparrow$, то и $g(x, y) \uparrow$. Пусть $f(x, y, a) \downarrow$. 
Рассмотрим два случая.

В первом случае $f(x, y, a) \in\{x, y\}$.

Полагаем $g(x, y)=f(x, y, a)$.

Во втором случае $f(x, y, a)=a$. Рассматриваем тот подслучай второго случая, выполнение условий которого выяснится раньше.

Подслучай $A$ : справедливо равенство $f(x, y, \bar{a})=x(f(x, y, \bar{a})=y)$. Полагаем $g(x, y)=y(g(x, y)=x)$, замечая, что $x, y \in A \Longrightarrow f(x, y, \bar{a}) \downarrow$.

Подслучай $B$ : найдется число $u$ такое, что $f(y, u, a)=u=f(x, u, \bar{a})$. Так как $u \in A \Longrightarrow x \in A$ и $u \in \bar{A} \Longrightarrow y \in \bar{A}$, полагаем $g(x, y)=x$.

Покажем, что если $A$ - не рекурсивно перечислимое множество (все рекурсивно перечислимые множества являются слабо $\beta$-комбинаторно селекторными множествами), $x \in A$ и $y \in \bar{A}$, то такое число $u$ существует. Рассмотрим рекурсивно перечислимые множества $B=\{u: f(x, u, a) \in\{a, u\}\}, C=\{u: f(x, u, \bar{a}) \in\{x, u\}\}$. Ясно, что $A \subseteq B \cap C$ и в качестве $u$ можно взять любое число из $(B \cap C) \backslash A$.

Подслучай $C$ : найдется число $u$ такое, что $f(x, u, a)=u=f(y, u, \bar{a})$. Полагаем $g(x, y)=y$.

Если же выполнение условий одного из подслучаев обнаружить не удается, то $g(x, y)$ неопределено.

\section{Список литературы}

1. Дегтев А. Н., Рекурсивно-комбинаторные свойства подмножеств натуральных чисел. Алгебра $u$ логика (1990) 29, №3, 303-314.

2. Дегтев А. Н., Импликативно селекторные множества. Алгебра и логика (1996) 35, №2, 145-153.

3. Дегтев А. Н., Иванов Д. И., Слабо комбинаторно селекторные множества. Алгебра и логика (1998) 37, №6, 627-636.

4. Дегтев А. Н., Иванов Д. И., Слабо импликативно селекторные множества размерности 3. Дискретная математика (1999) 11, №3, 126-132.

5. Дегтев А. Н., Почти полурекурсивные множества. Матем. заметки (1999) 66, №2, 188-193.

6. Дегтев А. Н., Почти комбинаторно селекторные множества. Матем. заметки (2000) 68, №6, 851-853.

7. Jockusch C. G., Semirecursive sets and positive reducibility. Trans. Amer. Math. Soc. (1968) 131, 420-436.

8. Jockusch C. G., Owings J. C., Weakly semirecursive sets. J. Symb. Logic (1990) 55, 627-644.

9. Kummer M., Stephan F., Weakly semirecursive sets and r. e. orderings. Ann. Pure Appl. Logic (1993) 60, 133-150. 